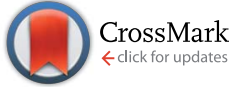

Cite this: RSC Adv., 2017, 7, 914

Received 27th October 2016 Accepted 14th November 2016

DOI: $10.1039 / c 6 r a 25906 e$

www.rsc.org/advances

\title{
Phototriggered base proliferation: a powerful 365 nm LED photoclick tool for nucleophile- initiated thiol-Michael addition reaction
}

\begin{abstract}
Ruixin Xu, $\uparrow^{a}$ Xiaoyuan Guan, $\dagger^{b}$ Minghui He $e^{* b}$ and Jianwen Yang ${ }^{c}$
Photocaged amines (PCAs) can allow a spatiotemporal control of the highly versatile and widely implemented nucleophile-catalyzed thiol-Michael addition reaction. However, a major challenge with most PCAs is that their relatively low quantum yields easily lead to low photosensitivity, especially under the radiation of light-emitting diodes (LEDs). In this paper, the phototriggered base proliferation (PBP) reaction as a powerful $365 \mathrm{~nm}$ LED photoclick tool is presented for the nucleophile-initiated thiolMichael addition reaction. Compared to the PCA system, the advantages of this approach lie in its enhanced photosensitivity, increased reaction rate and elevated conversion. Finally, due to the persistent interactions of the produced longeval amine, remarkable post conversion was thus initially achieved after irradiation.
\end{abstract}

\section{Introduction}

Over the past decade, click chemistry has continuously received a significant amount of attention in the design and synthesis of complex, highly functional molecules in areas such as polymer and material development, small molecule organic chemistry and drug discovery. ${ }^{1,2}$ One of the most attractive organic reactions, thiol-ene addition, has become popular owing to its ease of implementation, non-toxicity and high efficiency. ${ }^{3}$ Typically, thiol-ene addition reactions can proceed by radical-mediated anti-Markovnikov addition. The thiyl radical across the $\mathrm{C}=\mathrm{C}$ bond yields an intermediate carbon-centered radical, which can not only undergo the desired chain transfer reaction but also add another radical across the $\mathrm{C}=\mathrm{C}$ bond; i.e., the radical can propagate, resulting in oligomers/polymer as contaminants in the final product. It is obvious that one drawback of the radicalmediated thiol-ene addition reactions is the nonquantitative formation of target thioether with $\alpha, \beta$-unsaturated electrondeficient alkenes, such as acrylates and acrylamides. ${ }^{4}$

Much research has been directed toward the use of alternative amines or nucleophile-catalyzed Michael addition with quantitative conversions. ${ }^{4-10}$ To further achieve spatiotemporal control of this highly versatile and widely implemented coupling reaction, perhaps the most straightforward route is to

\footnotetext{
${ }^{a}$ School of Media and Communication, Shenzhen Polytechnic, Shenzhen, 518055, China

${ }^{b}$ State Key Laboratory of Pulp \& Paper Engineering, South China University of Technology, Guangzhou, 510640, China. E-mail: heminghui_2008@163.com

${ }^{\circ}$ DSAPM Lab, School of Materials Science and Engineering, Sun Yat-Sen University, Guangzhou, 510275, China

$\dagger$ These authors contributed equally to this work.
}

trigger the reaction by utilizing a photocaged amine (PCA). ${ }^{11-14}$ This spatiotemporal control can allow the on-demand initiation of a specific reaction, which is highly desirable under certain circumstances, and broaden its applications for the synthesis of functional materials. Due to the advantages of light-emitting diodes (LEDs), including lower costs, better environmentfriendliness and larger application areas, the photochemical reaction with LEDs has demonstrated enormous potential as a substitute for the traditional $\mathrm{Hg}$ lamp-induced reaction; ${ }^{15}$ however, classical PCAs usually suffer from poor light absorption properties for $\lambda \geq 365 \mathrm{~nm}$ because they were initially developed specifically for UV $\mathrm{Hg}$ lamps. Hence, a major challenge with most PCAs is that their relatively low quantum yields easily leads to low photosensitivity, especially under the radiation of LEDs.

In this context, the phototriggered base proliferation (PBP) reaction is expected to play a significant role in the thiolMichael addition reaction to improve the photo-initiated efficiency. The PBP reaction, which involved an autocatalytic decomposition of a base amplifier (BA) triggered by an amine catalyst from PCA, was pioneered by Ichimura and Arimitsu in $2000 .{ }^{16}$ The geometric progression of proliferated amine species causes a dramatic increase in the catalytic concentration and thus accelerates base-sensitive reactions. PBPs have been designed and applied in various types of photoreactive materials. ${ }^{17-20}$ Compared to PCA systems, this nonlinear organic reaction displays several hallmarks, such as high sensitivity, high quantum yield and short reaction times. By optimizing this unique proliferation characteristic, PBP reaction is transformed into a powerful and versatile methodology for nucleophile-initiated thiol-Michael addition photoclick reaction. 


\section{Experiments}

Materials

2-(2-Nitrophenyl)propyl chloroformate (NPPOC-Cl, 95\%, SigmaAldrich), 9-fluorenylmethyl chloroformate (Fmoc-Cl, 98\%, Shanghai Darui Finechem), hexylamine (HA, 98\%, Aladdinreagent), $\beta$-mercaptoethanol ( $\mathrm{ME},>98 \%$, Jian Yang Biotechnology), ethyl mercaptoacetate (EM, 98\%, Aladdin-reagent), hexanethiol (HE, 96\%, Aladdin-reagent), thioglycolic acid (TA, $\geq 98 \%$, Aladdin-reagent), benzyl mercaptan (BE, 99\%, Alfa Aesar), acrylic acid (AA, Tianjin Fu Chen Chemical Reagent Factory), and butyl acrylate (BA, 99\%, Aladdin-reagent) were used as received. All other chemicals used were of analytical grade and used without further purification.

\section{Methods}

The NMR spectra were obtained on a Varian $400 \mathrm{MHz}$ spectrometer with DMSO- $d_{6}$ and TMS as the solvent and internal standard, respectively. FTIR spectra were obtained over the wavenumber range of $400-4000 \mathrm{~cm}^{-1}$ on a Thermo Nicolet/ Nexus 670 spectrophotometer and recorded from 32 scans with a resolution of $4 \mathrm{~cm}^{-1}$. Real-time infrared spectroscopy (RTIR) was conducted to monitor the thiol-ene Michael addition reaction kinetics using a modified Nicolet 6700 (ThermoElectron, USA). UV-vis absorption spectra were obtained on a Perkin Elmer Lambda 750 UV-visible spectrophotometer. Electrospray ionization mass spectra (ESI-MS) were acquired on a Thermo Finnigan LCQ DECA XP ion trap mass spectrometer, equipped with an ESI source.

A UV-LED source (UVEC-4II, Lamplic Technology China) with a $365 \mathrm{~nm}$ emission wavelength was used for the photochemical reaction of the NPPOC-HA and PBP system with an irradiation intensity of $50 \mathrm{~mW} \mathrm{~cm}^{-2}$ (monitored by a radiometer, type UV-A, Photoelectric Instrument Factory, Beijing Normal University).

The resulting mixtures were uniformly smeared onto $\mathrm{KBr}$ crystals in a horizontal transmission apparatus, and then the reactions were initiated by the UV-LED source. Thiol conversion was monitored with the $\mathrm{S}-\mathrm{H}$ absorption peak at $2570 \mathrm{~cm}^{-1}$, and ene conversion was monitored with the $\mathrm{C}=\mathrm{C}$ absorption peak at $810 \mathrm{~cm}^{-1}$. Conversions were calculated by comparing the ratio of peak areas after the reaction to the peak areas prior to the reaction.

\section{Synthetic procedures and characterization}

Preparation of 2-(2-nitrophenyl)propyloxycarbonylhexylamine (NPPOC-HA). 2-(2-Nitrophenyl)propyl chloroformate (4 mmol, $0.9746 \mathrm{~g}$ ) was dissolved in $\mathrm{CH}_{2} \mathrm{Cl}_{2}(40 \mathrm{~mL})$, and then added dropwise to a solution of hexylamine (8.4 mmol, $0.8499 \mathrm{~g}$ ) in $\mathrm{CH}_{2} \mathrm{Cl}_{2}(20 \mathrm{~mL})$. After that, the mixture was stirred under cooling for $20 \mathrm{~min}$ and at room temperature for 5 hours. Finally, the solution was washed thrice successively with water, a $5 \% \mathrm{HCl}$ aqueous solution, and a saturated $\mathrm{NaCl}$ solution, dried over anhydrous $\mathrm{MgSO}_{4}$, and evaporated to obtain a light yellow liquid; then, the crude product was further purified by silica gel column chromatography using hexane : EtOAc $(10: 1)$ to obtain a pure light yellow oil as the product. Yield: $76 \% .{ }^{1} \mathrm{H} \mathrm{NMR}\left(400 \mathrm{MHz} \mathrm{CDCl}_{3}, \delta\right): 7.65(\mathrm{~d}, 1 \mathrm{H}$, H-a), 7.49 (t, 1H, H-b), 7.40 (d, 1H, H-c), 7.28 (t, 1H, H-d), 4.014.16 (m, 2H, H-e), 3.63 (t, 1H, H-f), 1.19-1.36 (t, 13H, H-g-i), 0.80 (t, 3H, H-h). ${ }^{13} \mathrm{CNMR}$ (400 MHz, $\mathrm{CDCl}_{3}, \delta$ ): 156.09, 150.77, $137.45,132.52,128.25,127.25,123.95,68.57,67.21,44.35$, $41.60,40.98,33.25,31.42,29.82,28.79,26.32,26.03,22.51$, 17.78, 17.32, 13.98 .

Preparation of 9-fluorenylmethoxycarbonyl-hexylamine (Fmoc-HA). Hexylamine (15.4 mmol, $1.561 \mathrm{~g})$ in $\mathrm{CH}_{2} \mathrm{Cl}_{2}(20$ $\mathrm{mL}$ ) was slowly added to an ice cooled solution of Fmoc-Cl (7.72 mmol, $2.015 \mathrm{~g}$ ) in $\mathrm{CH}_{2} \mathrm{Cl}_{2}(50 \mathrm{~mL})$, and then the mixture was stirred under cooling for $20 \mathrm{~min}$ and at room temperature for 1.5 hours. The solution was washed thrice successively with water, a $5 \% \mathrm{HCl}$ aqueous solution, and a saturated $\mathrm{NaCl}$ solution, dried over anhydrous $\mathrm{MgSO}_{4}$, and evaporated to obtain a solid; then, the solid was recrystallized from ethanol to obtain a white crystalline. Yield: $87 \%$. ${ }^{1} \mathrm{H}$ NMR (400 $\left.\mathrm{MHz} \mathrm{CDCl}_{3}, \delta\right)$ : 7.77 (d, 2H, H-a), 7.60 (d, 2H, H-b), 7.40 (t, 2H, H-c), 7.31 (t, 2H, $\mathrm{H}-\mathrm{d}$ ), 4.41 (d, 2H, H-e), 4.22 (t, 1H, H-f), 3.19 (m, 2H, H-g), 1.30 (m, 8H, H-h), 0.89 (t, 3H, H-i). ${ }^{13} \mathrm{CNMR}\left(400 \mathrm{MHz} \mathrm{CDCl}_{3}, \delta\right.$ ): 156.10, 144.56, 142.01, 128.81, 128.40, 126.78, 120.74, 66.98, $47.52,42.67,31.71,29.72,26.08,22.80,13.98$.

\section{Base proliferation in solution}

$n$-Hexylamine $\left(5 \mathrm{mmol} \mathrm{L}^{-1}\right)$ was added to a DMSO- $d_{6}$ solution of Fmoc-HA (100 $\left.\mathrm{mmol} \mathrm{L}^{-1}\right)$ in an NMR tube. The tube was sealed and subjected to real-time NMR measurement at $25{ }^{\circ} \mathrm{C}$. The proton of the methylene group was used to monitor the consumption of the Fmoc-HA.

\section{Results and discussion}

To develop the highly effective phototriggered base proliferation system, the highly active implementation presented in this paper has taken the following points into consideration. First, the photolytic deprotection reaction of the PCA should not generate any free radicals; the radical-mediated homopolymerization will reduce the ultimate conversion and thus affect the system's orthogonality and "click" nature. Moreover, thiols absorb light much more readily at lower wavelengths $(<320 \mathrm{~nm})$, and thus PCA should have relatively strong absorption above $320 \mathrm{~nm}$. In this paper, 2-(2-nitrophenyl)propyloxycarbonylhexylamine (NPPOC-HA) was synthesized by choosing the photolabile protecting group of 2-nitrobenzyl. Second, it should be noted that primary amines are more effective for the thiolMichael addition reaction than secondary amines. ${ }^{6}$ Beside the possessing lower volatility, the liberated amine from base amplifier should be strong enough to catalyze subsequent base proliferation reactions. Generally, the higher $\mathrm{p} K_{\mathrm{a}}$ of the proliferated amine resulted in a faster proliferation rate. Consequently, we designed 1-(9-fluorenylmethoxycarbonyl)-4hexylamine (Fmoc-HA) by utilizing the ideal $n$-hexylamine $\left(\mathrm{HA}, \mathrm{p} K_{\mathrm{a}}=10.56\right)$. The results from ${ }^{1} \mathrm{H}$ NMR, ${ }^{13} \mathrm{C}$ NMR and FTIR revealed that the targeted NPPOC-HA and Fmoc-HA were successfully obtained. 
The phototriggered base proliferation initiated thiol-Michael addition reaction is described in Fig. 1 , in which the regenerated $n$-hexylamine that is liberated from UV-irradiated NPPOC-HA and used as a phototrigger can promptly actuate highly effective base proliferation reactions; copious $n$-hexylamine species are thus generated such as in a domino reaction. Even under LED irradiation at a localized region, the regenerated $n$-hexylamine from NPPOC-HA can also diffuse into unirradiated regions and effectively actuate base proliferation reactions in three-dimensional spaces. The abruptly proliferated $n$-hexylamine molecules can be utilized to catalyze the thiol-Michael addition reaction, and this potentially results in enhanced photosensitivity and fast reaction rates. As a phototrigger, NPPOC-HA exhibits good absorption characteristics with a maximum at $198 \mathrm{~nm}$ and a tail above $300 \mathrm{~nm}$. It is obvious that NPPOC-HA has poor absorption, but it can also be used for UV $(>320 \mathrm{~nm})$ irradiation reactions. In particular, the extinction coefficient at $365 \mathrm{~nm}$ is $782 \mathrm{M}^{-1} \mathrm{~cm}^{-1}$.

ESI-MS was employed to detect the photodecomposed basic compounds included photogenerated $n$-hexylamine and proliferated $n$-hexylamine from $365 \mathrm{~nm}$ LED radiated PBP in DMSO. Before irradiation (Fig. 2a), signal molecules of $n$-hexylamine were not obtained in PBP solution. As shown in Fig. $2 \mathrm{~b}$, a tiny amount of protonated $n$-hexylamine $(m / z=102.3)$ was detected by ESI-MS after 30 min irradiation, which was direct evidence for the generation of $n$-hexylamine from irradiated NPPOC-HA. Furthermore, the protonated $n$-hexylamine $(\mathrm{m} / \mathrm{z}=102.3)$ was also successfully detected (Fig. 2c), and it is worth noting that the signal intensity of $n$-hexylamine ${ }^{+}$in Fig. $2 \mathrm{~b}$ is apparently smaller than Fig. 2c, as expected. These facts suggest that the highly effective base proliferation reaction can be readily actuated by employing NPPOC-HA as a phototrigger, and the massive $n$-hexylamine species can be further used to catalyze subsequent chemical reactions.

Prior to evaluating the PBP-initiated thiol-Michael addition photoclick reaction, base proliferation in solution was recorded by real-time ${ }^{1} \mathrm{H}$ NMR because the ability to initiate a rapid thiolMichael addition reaction is primarily dependent on the base proliferation rate. As shown in Fig. 3(1), there was no proton signal $\left(\mathrm{H}_{\mathrm{b}}, 6.25 \mathrm{ppm}\right)$ of vinyl found before the addition of hexylamine (Fig. 3(1)a), once the amine was added into the

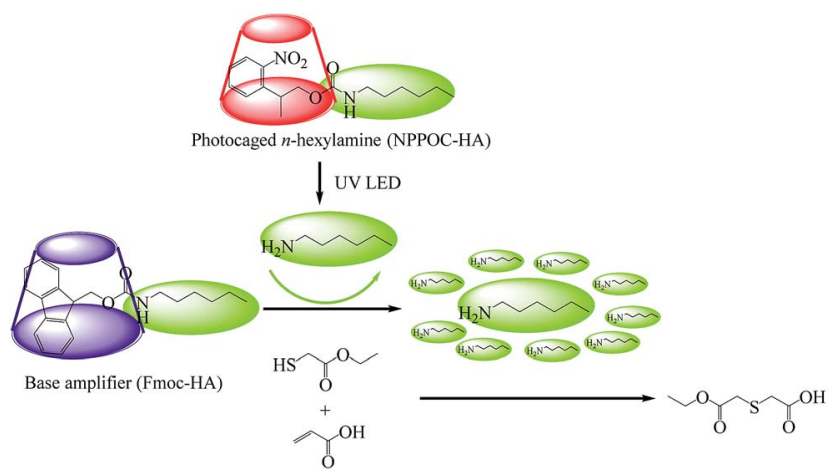

Fig. 1 Schematic illustration of phototriggered base proliferation initiated thiol-Michael addition reaction.

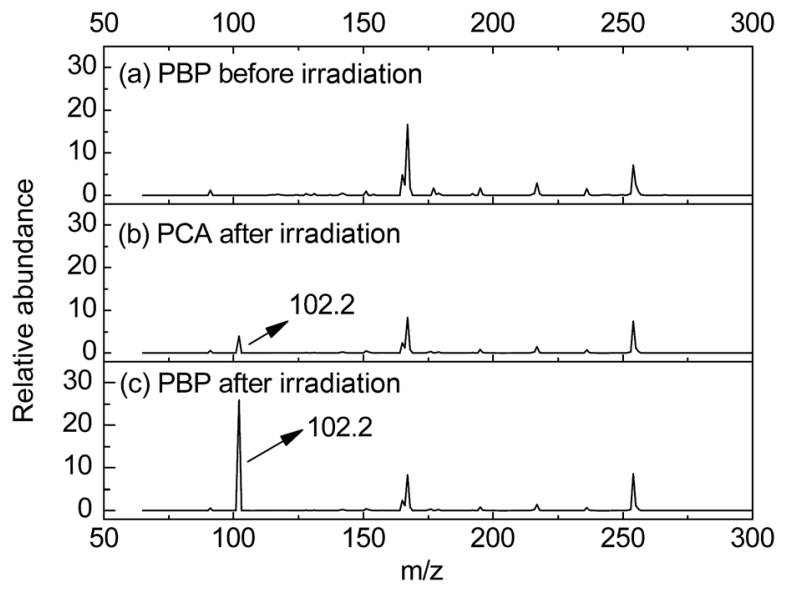

Fig. 2 Positive ion ESI-MS spectra (a) PBP before, (b) PCA after, and (c) PBP after $365 \mathrm{~nm}$ LED irradiation for $30 \mathrm{~min}$ in DMSO. Experimental conditions: PCA, [NPPOC-HA] $=0.01 \mathrm{mM}$; PBP, $[$ NPPOC-HA] $=$ $0.01 \mathrm{mM},[\mathrm{Fmoc}-\mathrm{HA}]=0.02 \mathrm{mM}$.

solution (Fig. 3(1)b), the proton signal $\left(\mathrm{H}_{\mathrm{b}}, 6.25 \mathrm{ppm}\right)$ of vinyl gradually increased as the reaction proceeded (Fig. 3(1)c). By monitoring the disappearing proton signals $\left(\mathrm{H}_{\mathrm{a}}, 4.23 \mathrm{ppm}\right)$ of
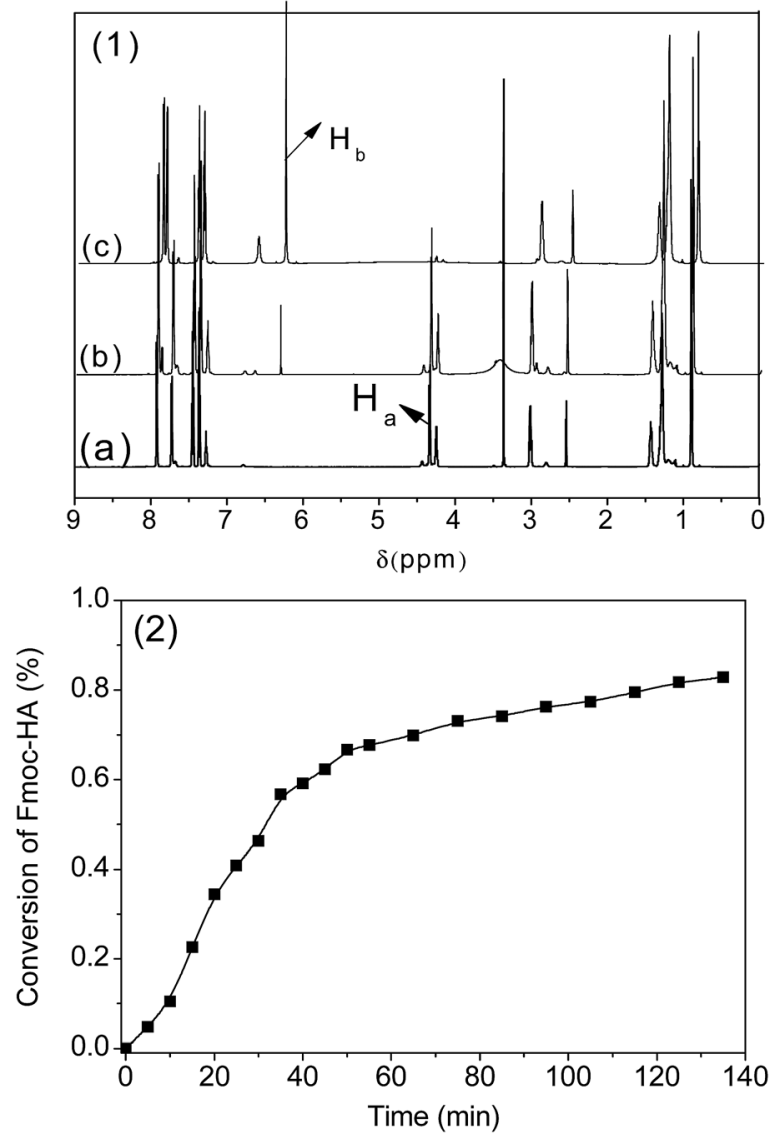

Fig. 3 (1) ${ }^{1} \mathrm{H}$ NMR spectral changes of Fmoc-HA in DMSO- $d_{6}$ $\left(100 \mathrm{mmol} \mathrm{L}^{-1}\right)(\mathrm{a})$ in the absence of and (b) in the presence of $n$ hexylamine $\left(5 \mathrm{mmol} \mathrm{L}^{-1}\right)$ at $25^{\circ} \mathrm{C}$ for $2 \mathrm{~min}$ and (c) for $120 \mathrm{~min}$; (2) the conversion of Fmoc-HA $\left(100 \mathrm{mmol} \mathrm{L}^{-1}\right)$ as a function of time in the presence of $n$-hexylamine $\left(5 \mathrm{mmol} \mathrm{L}^{-1}\right)$ in DMSO- $d_{6}$ at $25^{\circ} \mathrm{C}$. 
methylene and the DMSO- $d_{6}$ solvent peak at $2.5 \mathrm{ppm}$ as an internal standard based on ${ }^{1} \mathrm{H} \mathrm{NMR}$, and integrating the corresponding proton peak, the conversion curve in Fig. 3(1) was obtained. The approximately sigmoidal curve indicates that the decomposition of Fmoc-HA autocatalytically proceeds and lead to the proliferation of $n$-hexylamine at $25{ }^{\circ} \mathrm{C}$, and almost $70 \%$ conversion was obtained after $40 \mathrm{~min}$. Astonishingly, when the reaction temperature was increased to $80{ }^{\circ} \mathrm{C}$, the reaction profile was too fast to be measured by real-NMR, which indicated a complete reaction occurred in $<2 \mathrm{~min}$.

Next, real-time Fourier transform infrared (FTIR) spectroscopy was used to compare the catalytic behavior of PCA and PBP systems upon $365 \mathrm{~nm}$ LED irradiation at room temperature to demonstrate the potential of the two systems as photocatalysts for the thiol-Michael addition reaction; ethyl mercaptoacetate and acrylic acid were selected as model substrates. As shown in Fig. 4, these reactions could be rapidly actuated under photoirradiation without the presence of obvious induction periods, and the results of both PCA and PBP systems showed near complete conversions of both thiol and acrylate in $8 \mathrm{~min}$. As expected, under the same irradiation conditions, the system with a higher amine loading exhibited a faster reaction rate. In the case of the PCA system, an $80 \%$ thiol conversion required $3.44 \mathrm{~min}$, and the maximum initiation rate $\left(R_{\mathrm{pmax}}\right)$ was 49.4 $\mathrm{min}^{-1}$. Astonishingly, for the PBP system, the addition of FmocHA led to a sharp decrease of $T_{\mathrm{c} 80 \%}$ from $3.44 \mathrm{~min}$ to $2.38 \mathrm{~min}$ and a slight increase of $R_{\mathrm{pmax}}$ from $49.4 \mathrm{~min}^{-1}$ to $57.6 \mathrm{~min}^{-1}$; $80 \%$ thiol conversion was obtained in $1.21 \mathrm{~min}$. Moreover, the

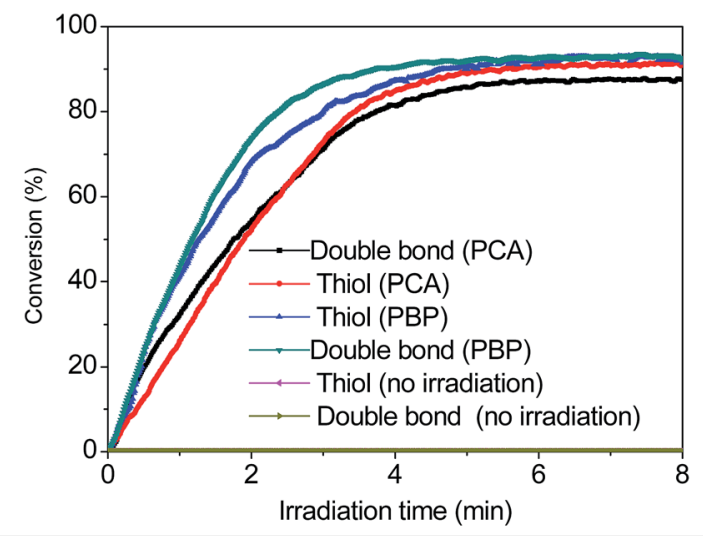

\begin{tabular}{lllll}
\hline System & Group & $\begin{array}{l}\mathrm{R}_{\mathrm{pmax}}{ }^{a} \\
(\% / \mathrm{min})\end{array}$ & $\begin{array}{l}\mathrm{T}_{\mathrm{c} 80 \%}{ }^{b} \\
(\mathrm{~min})\end{array}$ & $\begin{array}{l}\text { Final Conversion } \\
(\%)\end{array}$ \\
\hline \multirow{2}{*}{ PBG } & $\mathrm{C}=\mathrm{C}$ & 64.7 & 3.73 & 87 \\
& $\mathrm{SH}$ & 49.4 & 3.44 & 90 \\
\multirow{2}{*}{ PBA } & $\mathrm{C}=\mathrm{C}$ & 69.6 & 2.99 & 92 \\
& SH & 57.6 & 2.38 & 93
\end{tabular}

${ }^{a}$ The maximum initiation rate.

${ }^{\mathrm{b}} T_{C 80 \%}$ can be expressed by the reaction time at $80 \%$ conversion.

${ }^{\mathrm{c}}$ Irradiation time is $0 \mathrm{~min}$; reaction time is $30 \mathrm{~min}$.

Fig. 4 Conversion vs. time for the reaction for stoichiometric acrylic acid/ethyl mercaptoacetate mixtures upon irradiation by a $365 \mathrm{~nm}$ LED: (a) PCA system, [NPPOC-HA] $=0.16 \mathrm{mmol}$, (b) PBP system, [NPPOC-HA] : [Fmoc-HA] $=1: 1.5$, ethyl mercaptoacetate $(2 \mathrm{mmol})$, acrylic acid (2 $\mathrm{mmol})$ and butyl acrylate $(2 \mathrm{mmol})$.
PBP reaction without irradiation does not have a noticeable effect on the final conversion of thiol-Michael reaction. The faster photoefficiency of the PBP system can be attributed to the massive increase in the amount of amine catalysts, and this dramatically enhanced the reaction rate.

The adaptation of different monothiols catalyzed with PBP under the same $365 \mathrm{~nm}$ LED light source was critical in designing a highly effective base-catalyzed thiol-Michael addition photo-click reaction. In light of this, all of the monothiols in Fig. 5 were evaluated. Closer analysis of the data indicates that all monothiols obtained $>90 \%$ conversation, demonstrating that PBP can be utilized as a highly effective phototrigger for the base-catalyzed thiol-Michael photo-click reaction.

To further investigate the properties of the propagating reaction, we will address the need for the kinetic characterization of the $\mathrm{PBP}$ reaction by characterizing one of its distinguishing features: the long life-times of the active centers. Once the thiol-Michael addition reaction is initiated by the 365 LED light source, it continues after the light is shut off. As shown in Fig. 6, the sample illuminated for only $1 \mathrm{~min}$ continues to react for more than $11 \mathrm{~min}$ in the dark, and its final conversion rises to $\sim 77 \%$ from a threshold value of $\sim 40 \%$. The same final conversion value required a sustained illumination of $2.74 \mathrm{~min}$. This implies that enough active centers are

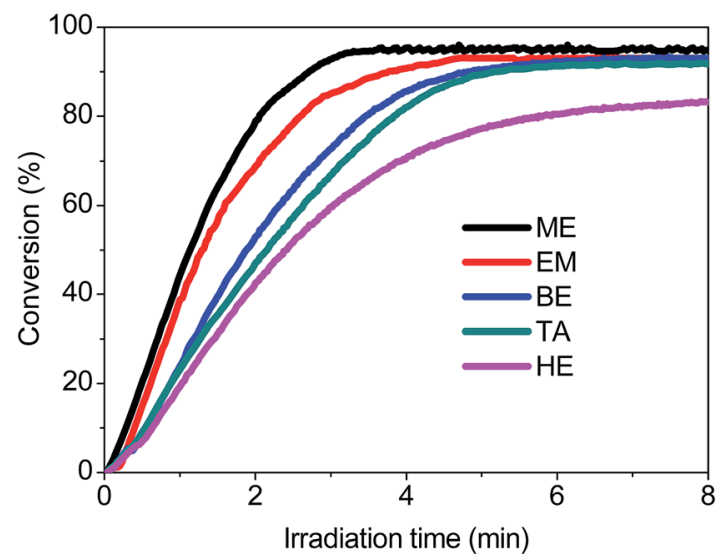

\begin{tabular}{lll}
\hline Entry & Thiol & $\begin{array}{l}\text { Irradiation time } \\
(\mathrm{min})\end{array}$
\end{tabular}

Fig. 5 Conversion vs. time for the reaction with different monothiols and acrylic acid catalyzed with the PBP system upon irradiation by a 365 nm LED: (a) PCA system, [NPPOC-HA] $=0.16 \mathrm{mmol}$, (b) PBP system, [NPPOC-HA] : [Fmoc-HA] $=1: 1.5$, monothiols $(2 \mathrm{mmol})$, acrylic acid $(2 \mathrm{mmol})$ and butyl acrylate $(2 \mathrm{mmol})$. 


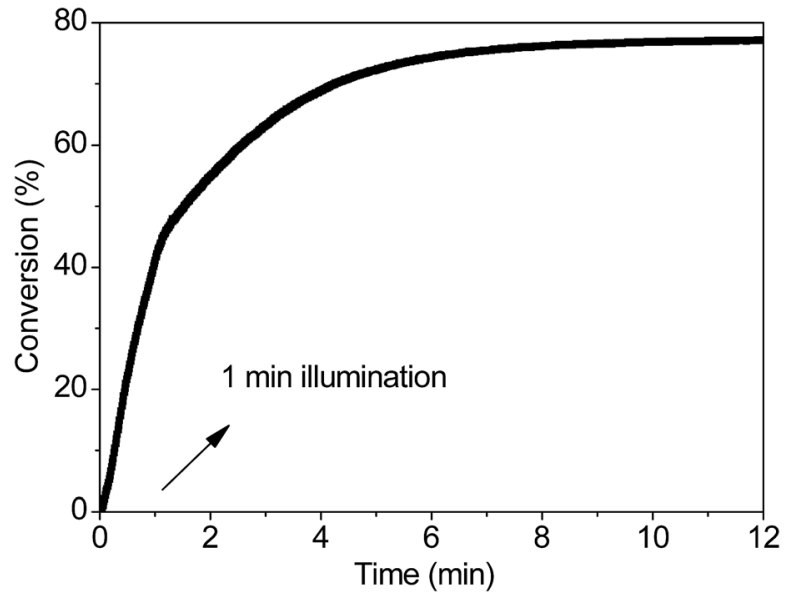

Fig. 6 Phototriggered propagating reaction after initial first $1 \mathrm{~min}$ illumination by 365 LED light source. Experimental conditions: $[\mathrm{NPPOC}-\mathrm{HA}]:[\mathrm{Fmoc}-\mathrm{HA}]=1: 1.5$, [NPPOC-HA] $=0.16 \mathrm{mmol}, \beta-$ mercaptoethanol (2 $\mathrm{mmol})$, ethyl mercaptoacetate $(2 \mathrm{mmol})$, acrylic acid $(2 \mathrm{mmol})$ and butyl acrylate $(2 \mathrm{mmol})$.

produced even after an illumination time as low as $1 \mathrm{~min}$; this is significant for the photo-screened reactions.

\section{Conclusions}

In conclusion, we have described a detailed study on the phototriggered base proliferation-induced thiol-Michael addition photo-click reaction as a powerful $365 \mathrm{~nm}$ LED photoclick tool. The results suggest that PBP offers a substantial breakthrough in the photoefficiency of amine generation, which results in a marked improvement in photosensitivity. Practically, this system was successfully used for the photo-screened reactions.

\section{Acknowledgements}

This research was financially supported by National Natural Science Foundation of China (21404042), Natural Science Foundation of Guangdong Province (2014A030310166), International Postdoctoral Exchange Fellowship Program ([2014] 29), Scientific Research Project of Shenzhen Polytechnic (601522k24011) and Fundamental Research Funds for the Central Universities (2016ZM060).

\section{Notes and references}

1 H. C. Kolb, M. G. Finn and K. B. Sharpless, Angew. Chem., Int. Ed., 2001, 40, 2004.
2 J. E. Moses and A. D. Moorhouse, Chem. Soc. Rev., 2007, 36, 1249.

3 C. E. Hoyle and C. N. Bowman, Angew. Chem., Int. Ed., 2010, 49, 1540.

4 J. W. Chan, C. E. Hoyle, A. B. Lowe and C. N. Bowman, Macromolecules, 2010, 43, 6381.

5 G. Z. Li, R. K. Randev, A. H. Soeriyadi, G. Rees, C. Boyer, Z. Tong, T. P. Davis, C. R. Becer and D. M. Haddleton, Polym. Chem., 2010, 1, 1196.

6 J. W. Chan, H. Wei, H. Zhou and C. E. Hoyle, Eur. Polym. J., 2009, 45, 2717.

7 O. Altintas, A. P. Vogt, C. Barner-Kowollik and U. Tunca, Polym. Chem., 2012, 3, 34.

8 D. P. Nair, N. B. Cramer, J. C. Gaipa, M. K. McBride, E. M. Matherly, R. R. McLeod, R. Shandas and C. N. Bowman, Adv. Funct. Mater., 2012, 22, 1502.

9 L. Maleki, U. Edlund and A. C. Albertsson, Biomacromolecules, 2015, 16, 667.

10 K. Jin, N. Wilmot, W. H. Heath and J. M. Torkelson, Macromolecules, 2016, 49, 4115.

11 W. Xi, M. Krieger, C. J. Kloxin and C. N. Bowman, Chem. Commun., 2013, 49, 4504.

12 S. Chatani, R. J. Sheridan, M. Podgórski, D. P. Nair and C. N. Bowman, Chem. Mater., 2013, 25, 3897.

13 W. Xi, H. Peng, A. Aguirre-Soto, C. J. Kloxin, J. W. Stansbury and C. N. Bowman, Macromolecules, 2014, 47, 6159.

14 X. Zhang, W. Xi, C. Wang, M. Podgórski and C. N. Bowman, ACS Macro Lett., 2016, 5, 229.

15 P. Xiao, J. Zhang, F. Dumur, M. A. Tehfe, F. Morlet-Savary, B. Graff, D. Gigmesb, J. P. Fouassierc and J. Lalevée, Prog. Polym. Sci., 2015, 41, 32.

16 K. Arimitsu, M. Miyamoto and K. Ichimura, Angew. Chem., Int. Ed., 2000, 39, 3425.

17 H. Mohapatra, K. M. Schmid and S. T. Phillips, Chem. Commun., 2012, 48, 3018.

18 T. Sasaki and H. Yaguchi, J. Polym. Sci., Part A: Polym. Chem., 2009, 47, 602.

19 (a) A. Igarashi, K. Arimitsu, T. Seki and K. Ichimura, J. Mater. Chem., 2008, 18, 560; (b) M. Furutani, H. Kobayashi, T. Gunji, Y. Abe and K. Arimitsu, J. Polym. Sci., Part A: Polym. Chem., 2015, 53, 1205; (c) K. Arimitsu, K. Tomota, S. Fuse, K. Kudo and M. Furutani, RSC Adv., 2016, 6, 38388; (d) K. Arimitsu, Y. Kumazawa and M. Furutani, J. Polym. Sci., Part A: Polym. Chem., 2015, 53, 2440; (e) K. Arimitsu, S. Amano and M. Furutani, RSC Adv., 2016, 6, 7893.

20 (a) M. He, X. Huang, Z. Zeng and J. Yang, Macromolecules, 2013, 46, 6402; (b) M. He, S. Jiang, R. Xu, J. Yang, Z. Zeng and G. Chen, J. Polym. Sci., Part A: Polym. Chem., 2014, 52, 1560 . 